\title{
The Instant and Sustained Effect of Electroacupuncture in Postgraduate Students with Depression: An fMRI Study
}

This article was published in the following Dove Press journal:

Neuropsychiatric Disease and Treatment

\section{Xiang-yu Wei (iD) \\ Hui Chen \\ Cui Guo \\ Wen-li Tan (D) \\ Song-hua Zhan (D)}

Department of Radiology, Shuguang Hospital Affiliated to Shanghai University of Traditional Chinese Medicine, Shanghai, 201203, People's Republic of China
Correspondence: Song-hua Zhan; Hui Chen Department of Radiology, Shuguang Hospital Affiliated to Shanghai University of

Traditional Chinese Medicine, 528

Zhangheng Road, Pudong New Area,

Shanghai, 201203, People's Republic of China

Email zhansonghua@sina.com;

chenhui660245@163.com
Objection: This study was a primary study to evaluate the instant and sustained effect of electroacupuncture (EA) at GV20 (Baihui) in postgraduate students with mild depression by using a special flexible head coil.

Methods: A total of 20 postgraduate students with mild depression underwent EA stimulation at GV20 and 3 phases of resting-state functional magnetic resonance imaging (rs-fMRI) scanning. Phase I: Preparation (before needle insertion); Phase II: during EA; Phase III: 15 minutes after needle removal. The Rs-fMRI data were processed using DPABI and SPSS 25. Results: 1) ReHo values showed significantly differences in the right posterior cingulate cortex, right calcarine gyrus, right angular gyrus, right precuneus, right cuneus, and bilateral postcentral gyri among Phase I, Phase II and Phase III; 2) Relative to the Phase I, increased brain activity in the Phase II was observed in the bilateral postcentral gyri, right calcarine gyrus, right cuneus. Compared with the Phase II, decreased brain activity in the Phase III was observed in the right precuneus, right posterior cingulate cortex, right angular gyrus. Relative to the Phase I, Significantly increased brain activity in the Phase III was observed in the right calcarine gyrus, right cuneus, and bilateral postcentral gyri. While decreased ReHo values were found in the right posterior cingulate cortex, right angular gyrus, right precuneus; and 3) Correlation analysis showed that the ReHo values of multiple brain regions in Phase I and Phase III were significantly correlated with the VAS and HRSD-17 scores.

Conclusion: This study focuses on the instant and sustained effect in postgraduate students with depression. Our study showed that instant effect produced by EA stimulation at GV20 firstly induced changes in somatosensory and visual area, and then, sustained effect (Phase III) have a higher intensity and more extensive than instant effects. Meanwhile, we provide a visualization way to study the instant effects of head acupoints by using a flexible head coil.

Keywords: acupuncture, functional magnetic resonance imaging; fMRI, depression, default mode network; DMN, neuroimaging

\section{Introduction}

A growing body of evidence suggests that people with higher education are more likely to suffer from depression and other mental disorders. ${ }^{1}$ The rising incidence of depression imposes a substantial burden on the group of postgraduate students and their family. Since the rise of acupuncture therapy, the clinical application for depression is very popular, many studies proved that acupuncture can relieve symptoms of depression. ${ }^{2}$ However, the instant effects and central treatment mechanism of acupuncture remain unclear. With the rapid development of resting- 
state functional magnetic resonance imaging (rs-fMRI), many studies have revealed various changes of function in brain regions of patients with depression. ${ }^{3}$ Recent data obtained by using rs-fMRI suggest that acupuncture has regionally specific effects on relevant brain structures and network activity. ${ }^{4}$

Previously, many acupuncture in depression researches came up with meaningful results. ${ }^{5,6}$ However, the acupoints and the acupuncture schemes in different studies vary greatly. ${ }^{7}$ Many acupoints for the treatment of depression are located in the head, such as Baihui (GV20), ${ }^{8}$ but synchronous rs-fMRI scanning is not feasible when acupuncture is done at the head acupoints. Moreover, rs-fMRI scanning are done after electroacupuncture (EA) in many studies, which may introduce several uncontrollable factors to the results and cannot reflect actual brain effect during EA. Meanwhile, previous research of head acupoints still limited by hardware, the instant effects on head acupoints have not been fully elucidated. It is difficult to collect the signal changes during the EA stimulation. $^{9}$

As mentioned above, there are many distractions in this non-real-time rs-fMRI approach. Therefore, we proposed a new concept of "real time rs-fMRI," a technology that can reflect complicated functional activities of brains instantly. We hypothesized that the instant and delayed brain effect of EA in depression was different. Our team have developed special flexible head coils successfully, which can collect rs-fMRI data during the head acupoints manipulation, and yields images of the same mass as the commercial head coil. Furthermore, the golden-needles, EA device in our study could compatible with the high magnetic field environment of MRI. Through the holes reserved in the flexible head coil, we can synchronize the head acupoints manipulation process and rs-fMRI scanning. At the same time, the EA device with magnetic shielding function can be placed beside the subjects, so that the change of subjects can be observed in real-time during the EA stimulation.

The head acupoints, including GV20, as widely used to treat mental disorders in Traditional Chinese Medicine (TCM). ${ }^{10}$ In the previous studies of acupuncture fMRI, the number of acupoints were not completely consistent. The changes of brain activity induced by acupoints combination could be overlapped or antagonized. ${ }^{11}$ Knowledge of the specific patterns of brain responses induced by stimulation at head acupoints remains limited. Therefore, starting from single acupoint was helpful to clarify the specificity of this acupoint. ${ }^{8}$

Regional homogeneity (ReHo) is a widely used method of rs-fMRI analysis, ${ }^{12,13}$ which is based on the principle of blood oxygen level dependence (BOLD) and reflects the synchronized neural activity functionally and commonly used in the study of depression, as well as other mental health disorders. ${ }^{14}$ Plenty of evidence has shown the abnormal ReHo value changes of brain areas in mental health disorders. ${ }^{15}$ To further investigate the similarities and differences between instant and sustained effects in ReHo values of depression, postgraduates with mild depression were recruited in this experiment and underwent acupuncture with GV20.

\section{Materials and Methods \\ Participants}

This study is quasi-experimental which can better control for confounding variables than other types of studies. The participants consisted of 22 Chinese postgraduate students with mild depression who were recruited from the Shanghai University of TCM from February to June 2020. Confirmation of the diagnosis of mild depression was made by two experienced psychiatrists using the structured Clinical Interview for DSM-IV (SCID). All subjects had normal structure examination results on MRI scan and signed a written informed consent form, as required by the ethics committee (Shuguang Hospital affiliated to Shanghai University of TCM, Approve Number: 2019-766121-01). The clinical trial registration number was ChiCTR1900023105. All participants included received a complete explanation of the study protocol. Their personal data were kept confidential and they were allowed to withdraw at any time during the quasi-experimental.

\section{Inclusion and Exclusion Criteria Inclusion Criteria}

1) all subjects were all at least 18 years old, right-handed, and were educated to postgraduate level; 2) all subjects had 7-24 points according to the HRSD-17; and 3) had not taken any analgesics, psychotropic drugs, antiinflammatory drugs or vasoactive drugs, and had not received any acupuncture treatment during the past 2 weeks before enrollment.

\section{Exclusion Criteria}

1) cannot stand acupuncture stimulation; 2) excessive head motion during MR scan; and 3) brain structure abnormality. 


\section{Acupuncture Manipulation}

EA was performed at GV20 acupoint and the EA stimulation paradigm used in this study was listed in detail (Figure 1). Equipment and devices used in this study were listed in Figure 2. Sterile non-magnetic 14K gold needle $(0.2 \times 35 \mathrm{~mm}$, Huatuo, Suzhou, China) was used. Considering the thin skin and lack of muscle tissue in the head, the tip of the needle was bent $15^{\circ}$ to the front to reach the aponeurosis cap with a depth of $20 \mathrm{~mm}$. The needle retained for 15 minutes and then the needle handle connected to low-frequency pulse therapy instrument (Huatuo brand, Model G6805-2, Suzhou China) through a special wire, set the continuous wave, frequency $2 \mathrm{~Hz}$, current $3 \mathrm{~mA}$, take the endurance of the subjects as the limit. The duration of EA was 5 minutes. All the procedures were performed by an experienced (more than 5 years) and licensed acupuncturist. When the experiment finished, the acupuncturist scored the acupuncture sensation of soreness by visual analogue scale (VAS).

\section{Rs-fMRI Scanning}

The Rs-fMRI scan including 3 phases, each with a duration time of 5 minutes, phase I: Preparation (before needle insertion); phase II: during EA stimulation; Phase III: The 15 minutes after EA stimulation. Rs-MRI images were acquired on a 3.0 Tesla UNITED IMAGING scanner (uMR780 platform, UNITED IMAGING Medical Systems, Shanghai, China) using 12 channel flexible head coil (Figure 2). A sponge-built head holder was used to prevent head movements. The parameters were as follows: 1) 3D-T1WI sequence structural imaging was performed with a T1weighted magnetization-prepared rapid gradient echo: $\mathrm{TR}=$ $10.5 \mathrm{~ms}, \quad \mathrm{TE}=4.4 \mathrm{~ms}, \mathrm{TI}=1120, \mathrm{FA}=15^{\circ}$, slice thickness $=1 \mathrm{~mm}, \mathrm{FOV}=330 \mathrm{~mm} \times 256 \mathrm{~mm}$, matrix $=256$ $\times 256$; and 2) Functional images were acquired with a singleshot gradient recalled echo planar imaging (EPI) sequence: $\mathrm{TR}=2000 \mathrm{~ms}, \mathrm{TE}=30 \mathrm{~ms}, \mathrm{FA}=90^{\circ}$, slice thickness $=3 \mathrm{~mm}$, $\mathrm{FOV}=240 \mathrm{~mm} \times 240 \mathrm{~mm}$, matrix $=64 \times 64,33$ slices. All subjects were keep their eyes closed and stay awake during the scanning process. They were also told to be relaxed and

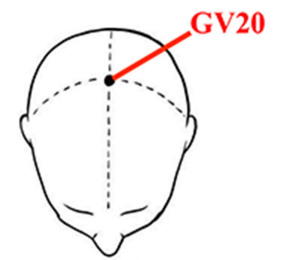

A

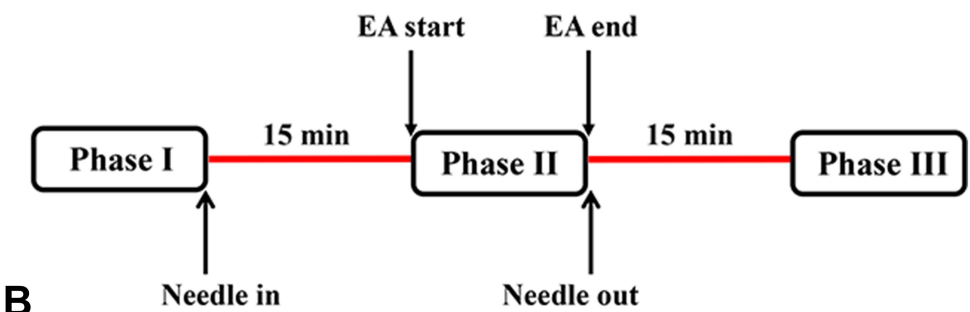

Figure I (A) The location of GV20 acupoint. (B) Paradigm of electro-acupuncture (EA) stimulation: the whole procedure lasted 40 min, consisting of a I5-minute acupuncture stimulation and three 5-minute MRI scans. Phase I: Preparation (before needle insertion), phase II: The first 5 minutes after initiation of EA stimulation, and Phase III: I5-20 minutes after needle removal. Needle in: The beginning of acupuncture at GV20. Needle out: needle removal. Abbreviation: EA, electro-acupuncture.

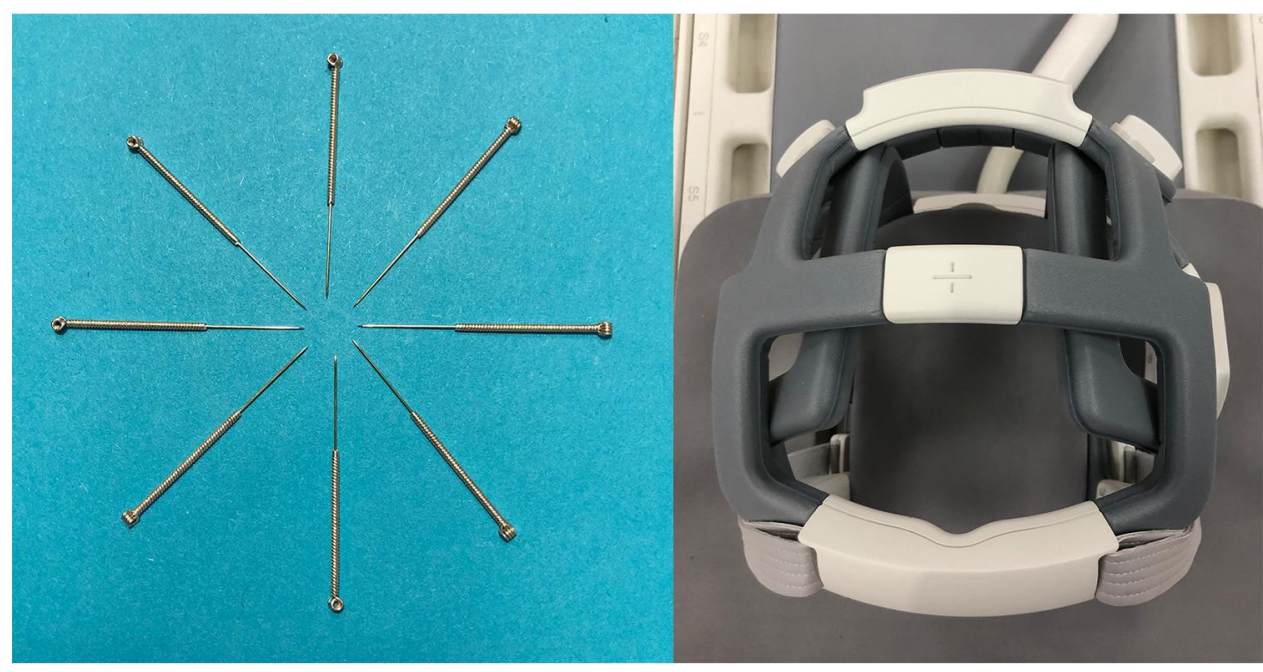

Figure 2 I4K gold needles (left panel) and flexible head coil (right panel). 
breathe peacefully with their heads fixed by foam headrests to reduce active or passive movements.

\section{Image Preprocessing}

Image preprocessing and $\mathrm{ReHo}^{12}$ analysis were conducted by Data Processing \& Analysis of Brain Imaging (DPABI, http://rfmri.org/dpabi). The first 10 volumes of functional time points that allowed the participants to adapt to scanning noises were discarded. The main steps included: 1) slicetiming; (2) realignment, the data at all time points were spatially aligned with the data collected at the first time point to obtain the head motion parameters of the subject in the scanning time series; 3) spatial normalization to the Montreal Neurological Institute (MNI) coordinate space with $3 \times 3 \times 3 \mathrm{~mm}^{3}$; 4) linear detrending; 5) a band-pass filtering (0.01-0.08 Hz); and 6) nuisance signals were regressed out, including Friston 24 head motion parameters, global signal, white matter signal, and cerebrospinal fluid signal.

Finally, the standardized ReHo map of each subjects were generated by calculating the Kendall Coefficient Concordance (KCC) of the time series of a given voxel with those of its nearest neighbors (27 voxels) in a voxel-wise manner. For subsequent statistical analysis, Fisher's r-to-z transformation was applied to improve the normality of the correlation. Then, a spatial smoothing with a $6 \mathrm{~mm}$ full width at half maximum (FWHM) isotropic Gaussian kernel was performed to reduce the noise of the normalized functional images.

\section{Statistical Analysis}

One-way ANOVA model was performed to compare whole brain ReHo to identify difference across 3 phases. The model was followed by post hoc $t$-tests to identify the differences between each pair of phases. In addition, all the comparisons were adjusted for head motion to control confounding effects. The brain areas first used voxel level correction, $p<0.001$, then FDR clusters level correction, cluster $>39$. Furthermore, Pearson correlation coefficients were computed between the extracted ReHo values within 3 phases and the clinical assessments, significance level was set at $p<0.05$ (two tailed).

The BrainNet Viewer (https://helab.bnu.edu.cn/brain net-viewer/) to show the results.

\section{Results}

\section{Demographic and Clinical Data (Table I)}

In this experiment, 2 subjects were excluded due to brain structure abnormality. Twenty subjects were finally included. All of them finished the EA stimulation and
Table I The Demographic and Clinical Data of Subjects $(n=20)$

\begin{tabular}{|l|c|}
\hline Characteristics & Frequency/Mean \pm SD \\
\hline Gender(male/female) & $10 / 10$ \\
Age(year) & $26.45 \pm 1.82(22-30)$ \\
Education(years) & $18.63 \pm 1.43(18-2 \mathrm{I})$ \\
HRSD-17 & $13.10 \pm 2.22(10-16)$ \\
Soreness(VAS) & $3.70 \pm 1.56(1-6)$ \\
\hline
\end{tabular}

Notes: Data are expressed as the mean \pm SD; Education, Transform the kind of education into the years of education; Primary school $\geq 5$ years, Middle school $\geq 6$ years, graduate $\geq 4$ years, Postgraduate $\geq 5$ years.

Abbreviation: HRSD-17, Hamilton Rating Scale for Depression (17 item version); VAS, Visual Analogue Scale.

fMRI scanning. No one dropped out of the experiment for excessive head motion and severe or unbearable pain.

\section{ReHo Results}

Significant difference in ReHo values were observed among 3 phase for the right posterior cingulate cortex, right calcarine gyrus, right angular gyrus, right precuneus, right cuneus, and bilateral postcentral gyri (Figure 3 and Table 2). Pairwise comparison results of ReHo values among 3 phases during fMRI scanning were shown in Figures 4-6 and Table 3. Specific comparisons between phases were: 1) The ReHo value in Phase II significantly increased compared to Phase I in the bilateral postcentral gyri, right calcarine gyrus, and right cuneus, while no significantly decreased brain was found (Figure 4); 2) The RhHo value in Phase III significantly decreased compared to Phase II in the right precuneus, posterior cingulate cortex, and right angular gyrus, while no significantly increased brain was found (Figure 5). and 3) ReHo value in Phase III significantly increased compared to Phase I in the right calcarine gyrus, right cuneus, and bilateral postcentral gyri, while decreased ReHo values were found in the right posterior cingulate cortex, right angular gyrus, and right precuneus (Figure 6).

\section{Correlation Between ReHo Values and Clinical Characteristics (Figure 7)}

The ReHo values of right postcentral gyri $(r=0.456$, $p=0.024)$ and left postcentral gyri $(r=0.590, p=0.009)$ in phase III was positively correlated with the VAS scores. The ReHo values of right posterior cingulate cortex $(r=-0.501$, $\mathrm{p}=0.024)$, right angular gyrus $(\mathrm{r}=-0.516, \mathrm{p}=0.02)$, right precuneus $(r=-0.501, p=0.024)$ in phase III was negatively correlated with VAS scores. The ReHo values of right postcentral gyri $(r=0.679, p=0.001)$ and left postcentral gyri $(r=0.618, p=0.004)$ in phase III was positively correlated 


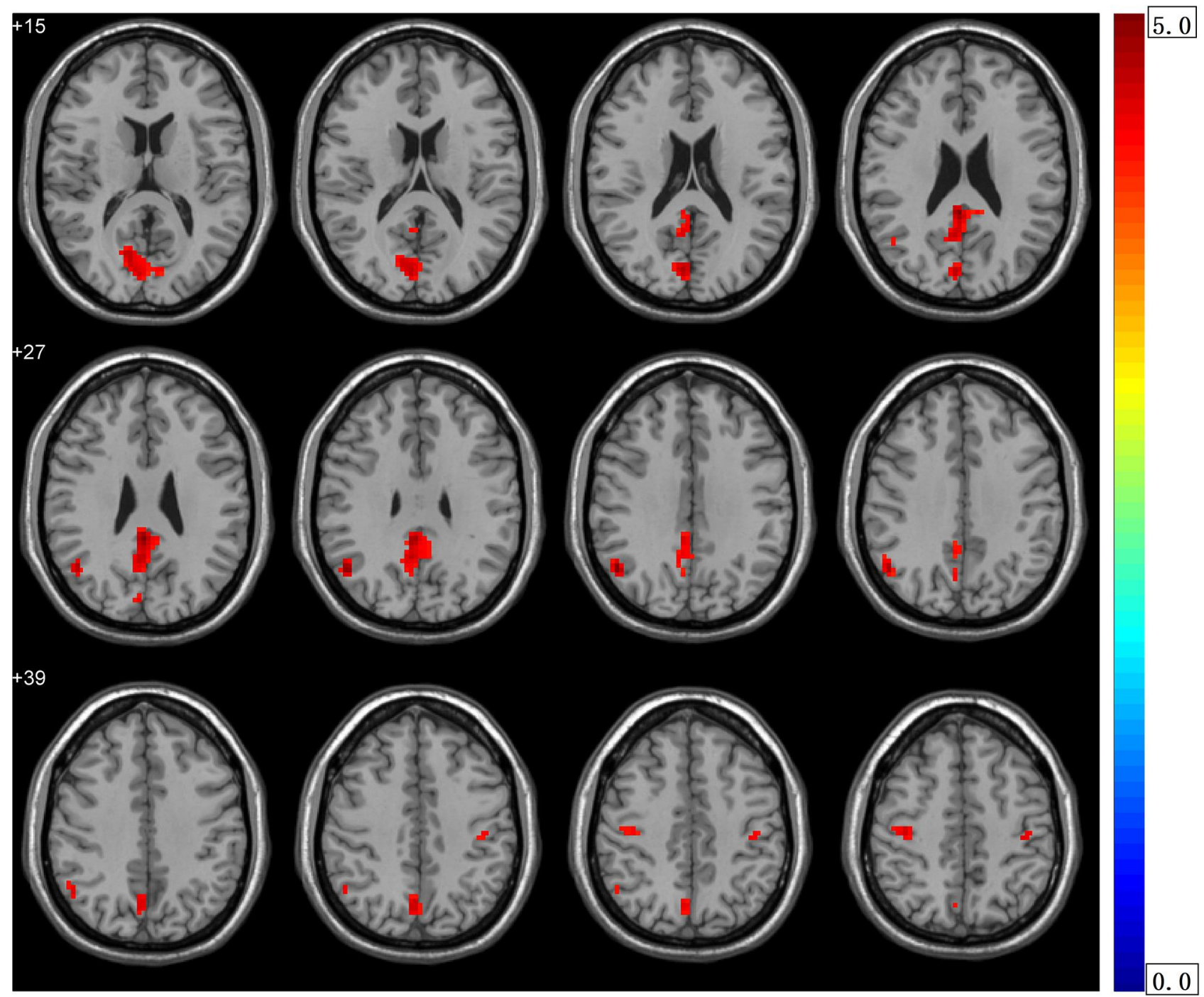

Figure 3 Comparison of ReHo values among the three phases. The red region in the figure shows significant difference in ReHo values among 3 phase, including the right posterior cingulate cortex, right calcarine gyrus, right angular gyrus, right precuneus, right cuneus, and bilateral postcentral gyri.

with the HRSD scores. The ReHo values of right angular gyrus $(r=0.447, p=0.048)$ in phase I was positively correlated with the HRSD scores.

\section{Discussion}

To our knowledge, this is the first study to explore the differences between instant and sustained effect of head acupoint in depression. Acupuncture treatment produces both instant and sustained effects, ${ }^{16-18}$ our study indicated that EA at GV20 could first instantly activated bilateral postcentral gyri, right calcarine gyrus, right cuneus. The bilateral postcentral gyri was the hub in the somatosensory network, which identified by early functional connectivity studies. ${ }^{19}$ The postcentral hub network had extended connectivity with primary sensory and motor cortices, which is consistent with the increased synchronization of neural activity in cortical regions during sensory processing, including a feel of pain, temperature, touch pressure, and so on. ${ }^{20}$ The existence of bilateral postcentral gyri in the somatosensory network suggests a stronger influence of the parallel architecture in this network. Apart from stronger activation in the bilateral postcentral gyri, we also find ReHo values increased in the right cuneus, calcarine gyrus. The cuneus hub was functionally connected to a visual network, and calcarine cortex as secondary hub of this network, these two hubs activated by tasks that require visual processing (i.e., visual attention, target detection, facial emotion recognition). ${ }^{21,22}$ The visual cortex is the initial stage of brain information reception and processing, is closely related to the further advanced processing of 
Table 2 ReHo Regions Differences in 3 Phases

\begin{tabular}{|c|c|c|c|c|c|c|c|}
\hline \multirow[t]{2}{*}{ Cluster } & \multicolumn{3}{|c|}{ MNI } & \multirow[t]{2}{*}{ F-value* } & \multicolumn{3}{|c|}{ Mean ReHo Value (Z-Transformed) } \\
\hline & $\mathbf{x}$ & $\mathbf{Y}$ & $\mathbf{Z}$ & & Phase I & Phase II & Phase III \\
\hline \multicolumn{8}{|c|}{ PCUN.R, extend to PCC.R } \\
\hline 182 & 3 & -42 & 27 & 29.25 & $1.585 \pm 0.055$ & $1.319 \pm 0.055$ & $0.991 \pm 0.055$ \\
\hline \multicolumn{8}{|c|}{ ANG.R } \\
\hline 44 & 51 & -63 & 33 & 29.06 & $1.524 \pm 0.049$ & $1.310 \pm 0.049$ & $0.996 \pm 0.049$ \\
\hline \multicolumn{8}{|c|}{ CAL.R, extend to CUN.R } \\
\hline 134 & 15 & -66 & 12 & 16.94 & $0.666 \pm 0.087$ & $1.094 \pm 0.087$ & $1.378 \pm 0.087$ \\
\hline \multicolumn{8}{|c|}{ PoCG.R } \\
\hline 63 & 45 & -18 & 57 & 14.35 & $0.226 \pm 0.087$ & $0.689 \pm 0.087$ & $0.864 \pm 0.087$ \\
\hline \multicolumn{8}{|c|}{ PoCG.L } \\
\hline 52 & -42 & -24 & 51 & 18.43 & $0.209 \pm 0.069$ & $0.635 \pm 0.069$ & $0.774 \pm 0.069$ \\
\hline
\end{tabular}

Notes: *Statistical significance level: first use voxel level correction, $p<0.00$ I, then FDR clusters level correction, cluster $>39$; Phase I, Preparation (before needle insertion); Phase II, during EA; Phase III, The 15 minutes after EA; X, Y, Z: coordinates of primary peak locations in the MNI space.

Abbreviations: L, left; R, right; MNI, Montreal Neurological Institute space; PCUN.R, right precuneus; PCC.R, right posterior cingulate cortex; ANG.R, right angular gyrus; CAL.R, right calcarine gyrus; CUN.R, right cuneus; PoCG.R, right postcentral gyri; PoCG.L, left postcentral gyri.

information (e.g., emotional processing, reward feedback, etc.). ${ }^{23,24} \mathrm{Li}$ et $\mathrm{al}^{25}$ found that, after $\mathrm{EA}$ stimulation, increased ReHo values were observed within the motor cortex and visual cortex. This result suggests that EA stimulation firstly induced changes in somatosensory and visual network, and indicates an important role of the postcentral, cuneus, calcarine gyrus hub in quickly sensory perception.

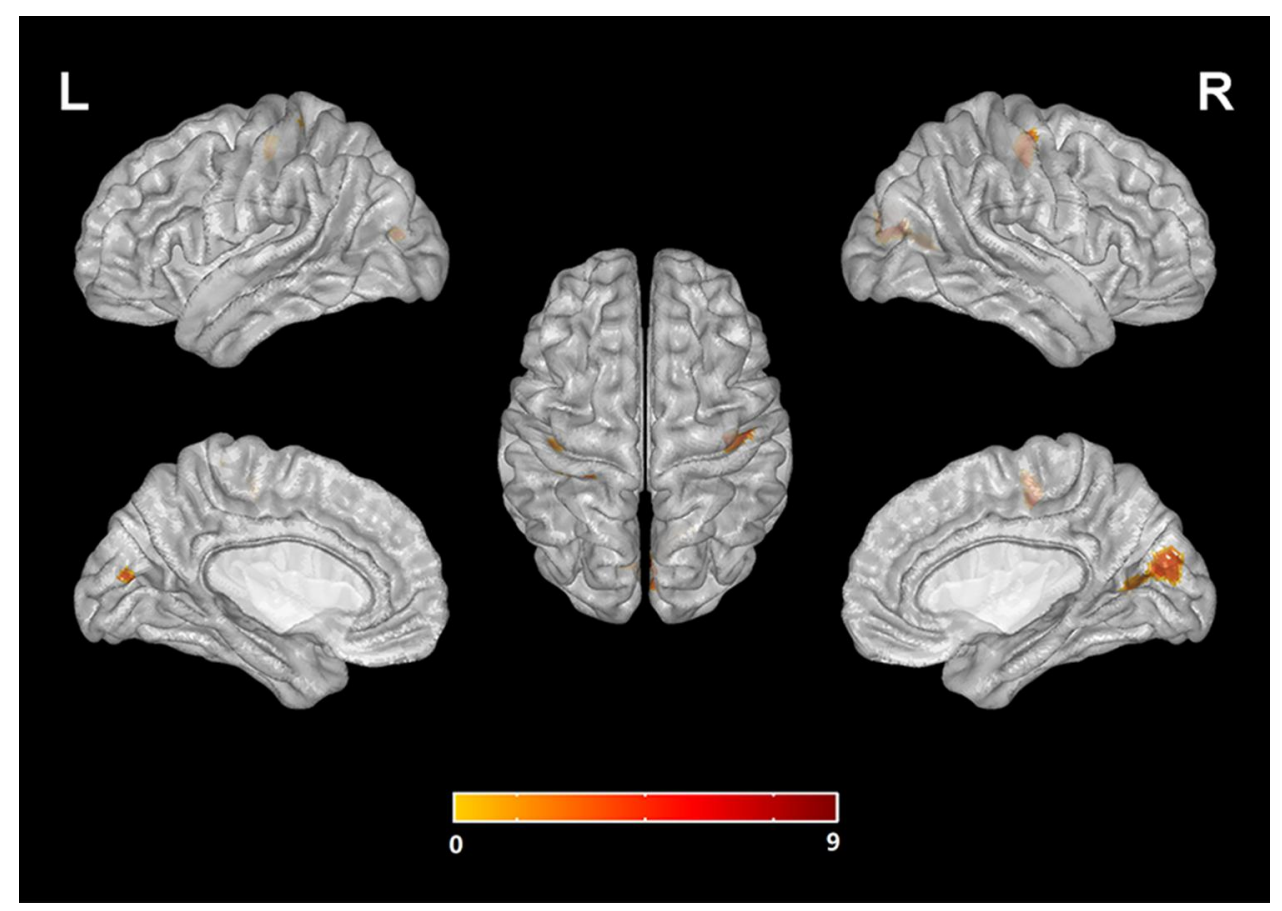

Figure 4 Pairwise comparison results of ReHo values between Phase II and Phase I. Brain regions with yellow-red colors indicate areas with a significantly increased ReHo value in Phase II compared with Phase I (a $\mathrm{p}<0.05$ with Bonferroni correction was adopted to correct for the number of tests). 


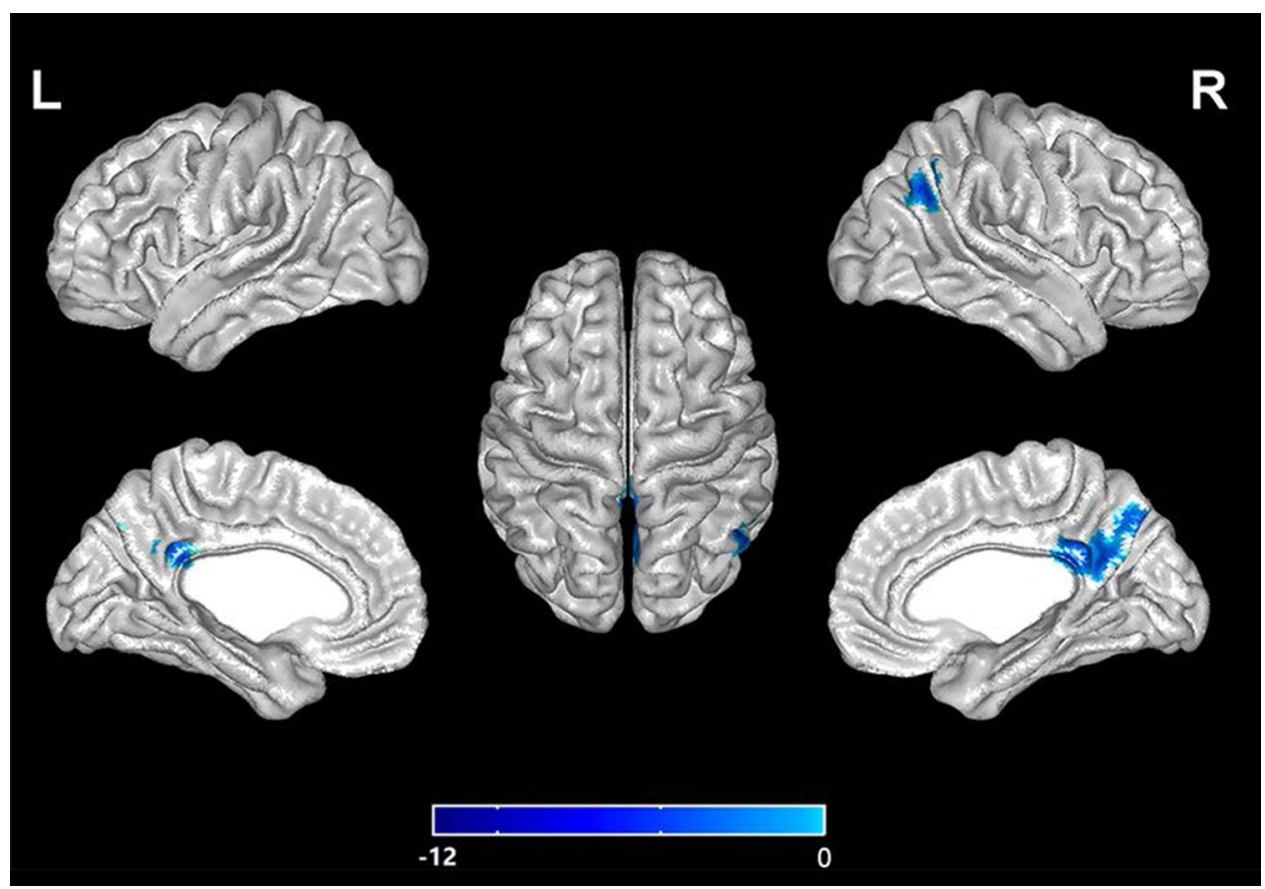

Figure 5 Pairwise comparison results of ReHo values between Phase III and Phase II. Brain regions with blue colors indicate areas with a significantly decreased ReHo value in Phase III compared with Phase II (a $\mathrm{P}<0.05$ with Bonferroni correction was adopted to correct for the number of tests).

Pairwise comparison between sustained effect (Phase III) and instant effect (Phase II) in EA stimulation found significantly decreased ReHo values in the posterior cingulate cortex, right angular gyrus, right precuneus. Interestingly, in previous research, posterior cingulate cortex and precuneus as the most connected hub was functionally linked to the default mode network (DMN) $)^{26,27}$ shows lower activity during goal-oriented task performance, ${ }^{28}$ which is consistent

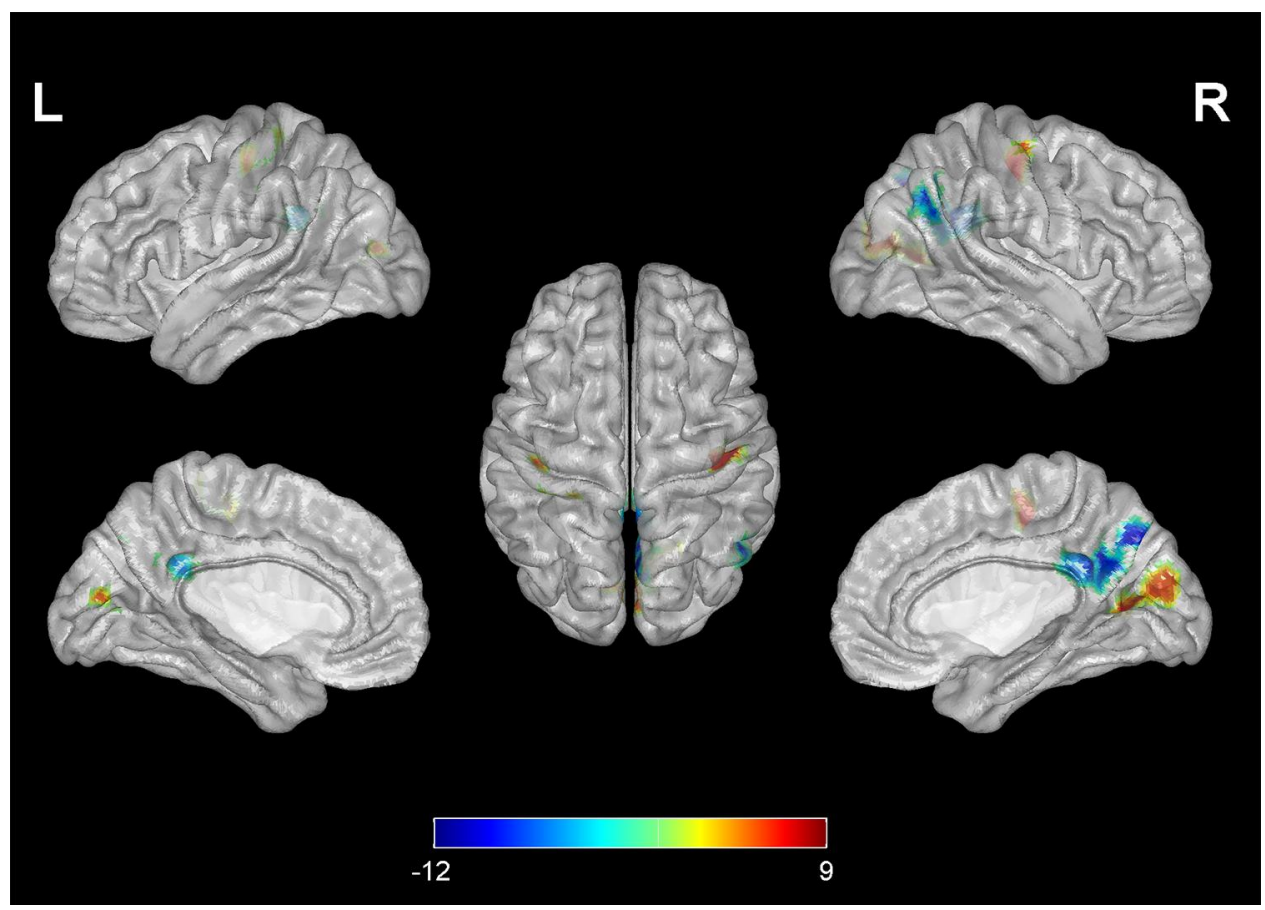

Figure 6 Pairwise comparison results of ReHo values between Phase III and Phase I. Brain regions with blue-green colors indicate areas with a significantly decreased ReHo value in Phase III compared with Phase I, while brain regions with yellow-red colors indicate areas a significantly increased ReHo value (a $\mathrm{P}<0.05$ with Bonferroni correction was adopted to correct for the number of tests). 
Table 3 Pairwise Comparison of ReHo Values of 3 Phases

\begin{tabular}{|c|c|c|}
\hline Pairwise Comparison & Mean Difference ReHo Value & p-value* \\
\hline \multicolumn{3}{|c|}{ PCUN.R, extend to PCC.R } \\
\hline Phase III < phase II & $-0.327 \pm 0.078$ & $<0.001$ \\
\hline Phase III < phase I & $-0.594 \pm 0.078$ & $<0.001$ \\
\hline \multicolumn{3}{|c|}{ ANG.R } \\
\hline Phase III < phase II & $-0.313 \pm 0.078$ & $<0.001$ \\
\hline Phase III < phase I & $-0.528 \pm 0.078$ & $<0.001$ \\
\hline \multicolumn{3}{|c|}{ CAL.R, extend to CUN.R } \\
\hline Phase II > phase I & $0.428 \pm 0.123$ & 0.003 \\
\hline Phase III > phase I & $0.712 \pm 0.123$ & $<0.001$ \\
\hline \multicolumn{3}{|c|}{ PoCG.R } \\
\hline Phase II > phase I & $0.463 \pm 0.123$ & 0.001 \\
\hline Phase III > phase I & $0.638 \pm 0.123$ & $<0.001$ \\
\hline \multicolumn{3}{|c|}{ PoCG.L } \\
\hline Phase II > phase I & $0.426 \pm 0.097$ & $<0.001$ \\
\hline Phase III > phase I & $0.565 \pm 0.097$ & $<0.001$ \\
\hline
\end{tabular}

Notes: Phase I, Preparation (before needle insertion); Phase II, during EA; Phase III, The 15 minutes after EA; Statistical significance level: $p<0.0033$ (a $p<0.05$ with Bonferroni correction was adopted to correct for the number of tests); *Stands for statistically significant.

Abbreviations: L, left; R, right; PCUN.R, right precuneus; PCC.R, right posterior cingulate cortex; ANG.R, right angular gyrus; CAL.R, right calcarine gyrus; CUN.R, right cuneus; PoCG.R, right postcentral gyri; PoCG.L, left postcentral gyri.

with our results. Deng et $\mathrm{al}^{29}$ found that after acupuncture stimulation at GV20, the abnormal DMN could be modulated, and angular gyrus located in the posterior part of the inferior parietal lobule, is also part of the DMN network. ${ }^{30}$ Depression is associated with abnormal function of the DMN, a distributed network of brain regions more active during rest than during performance of many tasks and characterized by a high degree of metabolic (i.e., posterior cingulate cortex, angular gyrus, and precuneus). The DMN is often found to be hyperactivated in depression. ${ }^{31}$ In this study, we found that ReHo value in Phase III significantly decreased in the right posterior cingulate cortex, right angular gyrus, and right precuneus. Compared with phase II, namely, during EA, these brain regions still show the characteristics of being suppressed, which means that the brain regions of DMN in depression was suppressed steadily during Phase III. We also found positive correlations between the HRSD-17 scores and the ReHo values of right angular gyrus in Phase I. The angular gyrus is involved in a wide array of cognitive functions including attention, episodic memory, conflict resolution, and action-outcome monitoring. ${ }^{32-34}$ The positive correlation between HRSD and right angular gyrus reflecting depression are associated with chronic stress and functional alterations in angular, which may account for the cognitive symptoms of depression, such as attention deficit, memory impairment, and negativity bias.

Since the DMN has been implicated in mind wandering, ${ }^{35}$ spontaneous processing, ${ }^{36}$ and performance of cognitive, ${ }^{37,38}$ we propose that the posterior cingulate cortex and precuneus hub performs information transfer and multimodal integration after EA stimulation, which might be produced after primary sensory and motor. Furthermore, compared with Phase II, our study indicated that the sustained effect produced by EA stimulation is stronger and more extensive than instant effect.

As another manifestation of the sustained effect, we noticed that more brain regions have positive or negative correlations with the VAS scores in phase III rather than Phase II. It may reflect the basic characteristics of EA and

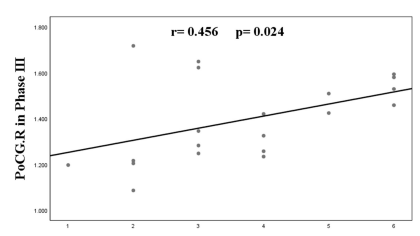

VAS

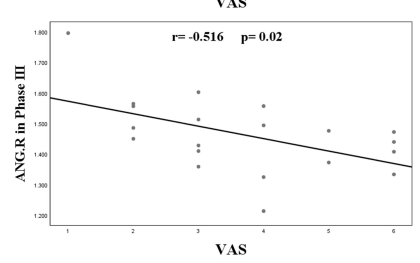

VAS

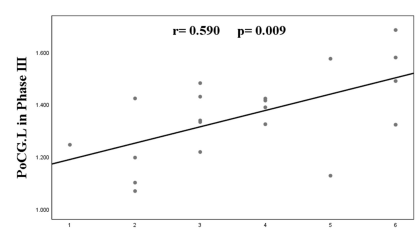

vaS

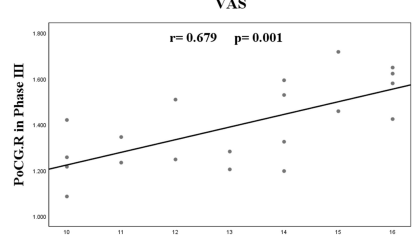

HRSD-17

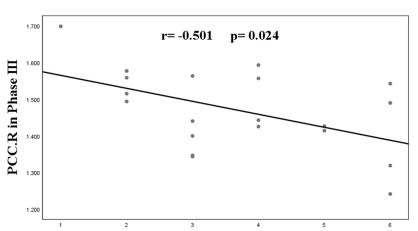

VAS

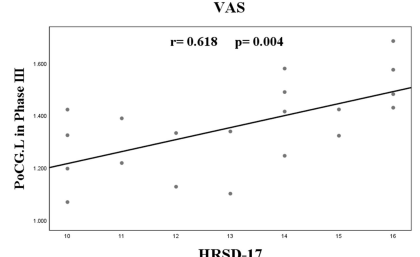

HRSD-17
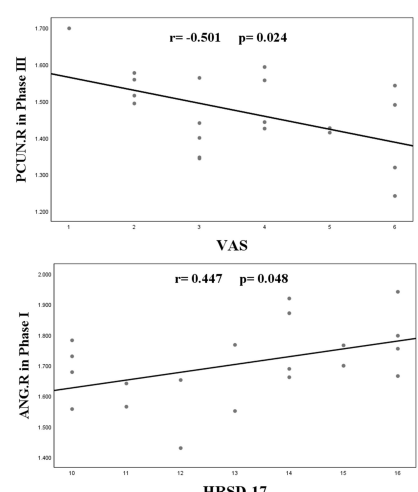

Figure 7 Correlation between ReHo values and clinical characteristics.

Abbreviations: PCUN.R, right precuneus; PCC.R, right posterior cingulate cortex; ANG.R, right angular gyrus; CAL.R, right calcarine gyrus; CUN.R, right cuneus; PoCG. R, right postcentral gyri; PoCG.L, left postcentral gyri; HRSD-17, Hamilton Rating Scale for Depression (I7 item version); VAS, Visual Analogue Scale. 
the response speed of depression patients to pain. We speculated that the VAS scores was more related to the sustained effect, namely, phase III than instant effect, which may related to the insensitivity or self-adaptation to pain under depression. The time-dependence effect are consistent with the brain's processing of pain produced by EA stimulation. However, previous results on the perception of EA-induced pain in depression showing both an increased and decreased pain threshold and pain tolerance across various studies. ${ }^{39,40}$ Therefore, further study is needed to clarify the relationship between depression and pain. This study provide neuroimaging basis for the real time effect of EA stimulation at GV20 in mild depression.

In commercial head coil, the situation of GV20 acupoints was covered by the coil and could not be penetrated by the acupuncturist. Our team has designed a flexible head coil to solve this problem. To ensure the imaging quality and improve the signal-to-noise ratio (SNR) of the image, the coil was designed to be adjustable, so that the coil can closely fit the head of the subjects. Compared with 16 channels hard coil, the SNR of the image was higher in our 12 channels flexible head coil. ${ }^{41}$ In our experimental design, after needle removal, we kept the subjects continue to remain rest state for 15 minutes. With the help of flexible head coil, the subjects could perform the third phase of fMRI scan without any activity, the subjects' status remained consistent as much as possible during the 3 phases. Therefore, in this experiment, time is the main factor affecting the change of brain effect, avoid the interference of extra activities of subjects.

Several limitations of this study should be addressed. First, the relatively modest patient sample size is a limitation of our study, and we cannot rule out the possibility that some of the changes we observed after EA were not causally related to depression. More subjects need to be included for further analysis. Second, the crosssectional nature of this study necessitates future longitudinal studies to evaluate the sensitivity of ReHo analyses in predicting progression of depression. Third, the observation period after needle removal in this study was too short for further investigation on delayed changes in brain regions. Thus, further studies with a larger sample size, inclusion of both healthy and diseased population, and longer observation time are warranted to determine the efficacy, underlying neural mechanism, and long-term outcome of EA stimulation at GV20 in a more conclusive fashion. At the same time, we need to continue to explore new scanning methods and experimental design ideas.

\section{Conclusions}

This is the first report to observe both instant and sustained effect of EA at GV20 in depression. Changes of brain function during different phases of depression were studied in detail. The results indicate that the sustained effect produced by EA suppresses hyperactivated of DMN in depression. The sustained effect is stronger, more extensive than instant effect and triggers a brain response leading to the integration of a cerebral network in depression, involving both sensation and perception. At the same time, we should note that the conclusions in our study may not apply to all types of depression (e.g., major depressive disorder) andmore study in the same field is needed.

\section{Data Sharing Statement}

The data which support the conclusions of our study is included within the article.

\section{Ethical Approval}

The study compliances with the ethical standards of the responsible committee on human experimentation (Shuguang Hospital, Shanghai University of Traditional Chinese Medicine, Approve Number: 2019-766121-01) and with the Declaration of Helsinki (1964).

\section{Author Contributions}

All authors made substantial contributions to conception and design, acquisition of data, or analysis and interpretation of data; took part in drafting the article or revising it critically for important intellectual content; agreed to submit to the current journal; gave final approval of the version to be published; and agree to be accountable for all aspects of the work.

\section{Funding}

This research was supported by:National Natural Science Foundation of China (No. 81573782); 2017 Shanghai University of Traditional Chinese Medicine Major Achievement Project (ZDCG201701); Shuguang Hospital affiliated to Shanghai University of Traditional Chinese Medicine, National Natural Science Foundation Incubation Project (No. SGYYYX-202001). The sponsor provided financial support in the case observation, the collection, analysis of data, and the decision to submit the article for publication.

\section{Disclosure}

The authors report no conflicts of interest for this work and declare that they have no known competing financial 
interests or personal relationships that could have appeared to influence the work reported in this article.

\section{References}

1. Rotenstein LS, Ramos MA, Torre M, et al. Prevalence of depression, depressive symptoms, and suicidal ideation among medical students: a systematic review and meta-analysis. JAMA. 2016;316 (21):2214-2236. doi:10.1001/jama.2016.17324

2. Li M, Niu J, Yan P, et al. The effectiveness and safety of acupuncture for depression: an overview of meta-analyses. Complement Ther Med. 2020;50:102202. doi:10.1016/j.ctim.2019.102202

3. Li Z, Zeng F, Yin T, et al. Acupuncture modulates the abnormal brainstem activity in migraine without aura patients. Neuroimage Clin. 2017;15:367-375. doi:10.1016/j.nicl.2017.05.013

4. Liang P, Wang Z, Qian T, Li K. Acupuncture stimulation of Taichong (Liv3) and Hegu (LI4) modulates the default mode network activity in Alzheimer's disease. Am J Alzheimers Dis Other Demen. 2014;29 (8):739-748. doi:10.1177/1533317514536600

5. Fang J, Rong P, Hong Y, et al. Transcutaneous vagus nerve stimulation modulates default mode network in major depressive disorder. Biol Psychiatry. 2016;79(4):266-273. doi:10.1016/j.biopsych.2015.03.025

6. Wang Z, Wang X, Liu J, et al. Acupuncture treatment modulates the corticostriatal reward circuitry in major depressive disorder. $J$ Psychiatr Res. 2017;84:18-26. doi:10.1016/j.jpsychires.2016.09.014

7. Sallach K, Leonhardt M. Akupunktur zur Behandlung depressiver Störungen bei Schmerzerkrankungen [Acupuncture for treatment of depressive disorders in pain diseases]. Der Nervenarzt. 2018;89 (9):986-993. German. doi:10.1007/s00115-018-0576-3

8. Mao L, Lv FF, Yang WF, et al. Effects of Baihui electroacupuncture in a rat model of depression. Ann Transl Med. 2020;8(24):1646. doi:10.21037/atm-20-7459

9. Guo J, Chen Y, Li Z, et al. The cerebral mechanism of acupuncture for treating knee osteoarthritis: study protocol for a randomized controlled trial. Trials. 2019;20(1):126. doi:10.1186/s13063-019-3233-7

10. Su KQ, Liu ST, Li JY, et al. Effects of different acupuncture treatment methods on post-stroke cognitive impairment: study protocol for a multicenter randomized controlled trial. Trials. 2021;22(1):27. doi:10.1186/s13063-020-04959-y

11. Cai RL, Shen GM, Wang H, Guan YY. Brain functional connectivity network studies of acupuncture: a systematic review on resting-state fMRI. J Integr Med. 2018;16(1):26-33. doi:10.1016/j.joim.2017.12.002

12. Zang Y, Jiang T, Lu Y, et al. Regional homogeneity approach to fMRI data analysis. Neuroimage. 2004;22(1):394-400. doi:10.1016/j. neuroimage.2003.12.030

13. Zuo XN, Xu T, Jiang L, et al. Toward reliable characterization of functional homogeneity in the human brain: preprocessing, scan duration, imaging resolution and computational space. Neuroimage. 2013;65:374-386. doi:10.1016/j.neuroimage.2012.10.017

14. Liu P, Li Q, Zhang A, et al. Similar and different regional homogeneity changes between bipolar disorder and unipolar depression: a resting-state fMRI study. Neuropsychiatr Dis Treat. 2020;16:1087-1093. doi:10.2147/NDT.S249489

15. Liang MJ, Zhou Q, Yang KR, et al. Identify changes of brain regional homogeneity in bipolar disorder and unipolar depression using resting-state FMRI. PLoS One. 2013;8(12):e79999. doi:10.1371/journal.pone.0079999

16. Li J, Ran X, Cui C, et al. Instant sedative effect of acupuncture at GV20 on the frequency of electroencephalogram alpha and beta waves in a model of sleep deprivation. Exp Ther Med. 2018;15 (6):5353-5358. doi:10.3892/etm.2018.6123

17. Jin $\mathrm{L}$, Sun $\mathrm{J}, \mathrm{Xu} \mathrm{Z}$, et al. Intersubject synchronisation analysis of brain activity associated with the instant effects of acupuncture: an fMRI study. Acupunct Med. 2018;36(1):14-20. doi:10.1136/ acupmed-2016-011327
18. Long X, Huang W, Napadow V, et al. Sustained effects of acupuncture stimulation investigated with centrality mapping analysis. Front Hum Neurosci. 2016;10:510. doi:10.3389/fnhum.2016.00510

19. Xiong J, Parsons LM, Gao JH, Fox PT. Interregional connectivity to primary motor cortex revealed using MRI resting state images. Hum Brain Mapp. 1999;8(2-3):151-156. doi:10.1002/(sici)1097-0193(1999)8:2/3<151::aid-hbm13>3.0.co;2-5

20. Srinivasan R, Russell DP, Edelman GM, Tononi G. Increased synchronization of neuromagnetic responses during conscious perception. $J$ Neurosci. 1999;19(13):5435-5448. doi:10.1523/ JNEUROSCI.19-13-05435.1999

21. Tomasi D, Volkow ND. Association between functional connectivity hubs and brain networks. Cereb Cortex. 2011;21(9):2003-2013. doi:10.1093/cercor/bhq268

22. Kong J, Kaptchuk TJ, Webb JM, et al. Functional neuroanatomical investigation of vision-related acupuncture point specificity-a multisession fMRI study. Hum Brain Mapp. 2009;30(1):38-46. doi:10.1002/hbm.20481

23. Chen C, Jiang WH, Wang W, et al. Impaired visual, working, and verbal memory in first-episode, drug-naive patients with major depressive disorder in a Chinese population. PLoS One. 2018;13(4): e196023. doi:10.1371/journal.pone.0196023

24. Guo W, Liu F, Xue Z, et al. Abnormal resting-state cerebellar-cerebral functional connectivity in treatment-resistant depression and treatment sensitive depression. Prog Neuropsychopharmacol Biol Psychiatry. 2013;44:51-57. doi:10.1016/j.pnpbp.2013.01.010

25. Li Z, Chen J, Cheng J, et al. Acupuncture modulates the cerebellothalamo-cortical circuit and cognitive brain regions in patients of Parkinson's disease with tremor. Front Aging Neurosci. 2018;10:206. doi:10.3389/fnagi.2018.00206

26. Buckner RL, Sepulcre J, Talukdar T, et al. Cortical hubs revealed by intrinsic functional connectivity: mapping, assessment of stability, and relation to Alzheimer's disease. J Neurosci. 2009;29 (6):1860-1873. doi:10.1523/JNEUROSCI.5062-08.2009

27. Sarubbo S, Tate M, De Benedictis A, et al. Mapping critical cortical hubs and white matter pathways by direct electrical stimulation: an original functional atlas of the human brain. Neuroimage. 2020;205:116237. doi:10.1016/j.neuroimage.2019.116237

28. Larabi DI, Renken RJ, Cabral J, et al. Trait self-reflectiveness relates to time-varying dynamics of resting state functional connectivity and underlying structural connectomes: role of the default mode network. Neuroimage. 2020;219:116896. doi:10.1016/j.neuroimage.2020.116896

29. Deng D, Liao H, Duan G, et al. Modulation of the default mode network in first-episode, drug-naive major depressive disorder via acupuncture at Baihui (GV20) acupoint. Front Hum Neurosci. 2016;10:230. doi:10.3389/fnhum.2016.00230

30. Zhang R, Volkow ND. Brain default-mode network dysfunction in addiction. Neuroimage. 2019;200:313-331. doi:10.1016/j.neuroimage.2 019.06.036

31. Whitfield-Gabrieli S, Ford JM. Default mode network activity and connectivity in psychopathology. Annu Rev Clin Psychol. 2012;8:49-76. doi:10.1146/annurev-clinpsy-032511-143049

32. Wei Y, Bresser T, Wassing R, et al. Brain structural connectivity network alterations in insomnia disorder reveal a central role of the right angular gyrus. Neuroimage Clin. 2019;24:102019. doi:10.1016/ j.nicl.2019.102019

33. van Kemenade BM, Arikan BE, Kircher T, Straube B. The angular gyrus is a supramodal comparator area in action-outcome monitoring. Brain Struct Funct. 2017;222(8):3691-3703. doi:10.1007/s00429017-1428-9

34. Ramanan S, Piguet O, Irish M. Rethinking the role of the angular gyrus in remembering the past and imagining the future: the contextual integration model. Neuroscientist. 2018;24(4):342-352. doi: $10.1177 / 1073858417735514$ 
35. Zanao TA, Lopes TM, de Campos BM, et al. Patterns of default mode network in temporal lobe epilepsy with and without hippocampal sclerosis. Epilepsy Behav. 2019:106523. doi:10.1016/j.yebeh.2019.106523

36. Alshelh Z, Marciszewski KK, Akhter R, et al. Disruption of default mode network dynamics in acute and chronic pain states. Neuroimage Clin. 2018;17:222-231. doi:10.1016/j.nicl.2017.10.019

37. Zhang G, Zhang H, Li X, et al. Functional alteration of the DMN by learned regulation of the PCC using real-time fMRI. IEEE Trans Neural Syst Rehabil Eng. 2013;21(4):595-606. doi:10.1109/ TNSRE.2012.2221480

38. Scheffold BE, Hsieh CL, Litscher G. Neuroimaging and neuromonitoring effects of electro and manual acupuncture on the central nervous system: a literature review and analysis. Evid Based Complement Alternat Med. 2015;2015:641742. doi:10.1155/2015/641742
39. Nitzan U, Hecht M, Braw Y, et al. Initial evaluation of pain intensity among depressed patients as a possible mediator between depression and pain complaints. Front Psychiatry. 2019;10:48. doi:10.3389 fpsyt.2019.00048

40. Rodriguez-Raecke R, Ihle K, Ritter C, et al. Neuronal differences between chronic low back pain and depression regarding long-term habituation to pain. Eur J Pain. 2014;18(5):701-711. doi:10.1002/ j.1532-2149.2013.00407.x

41. Gong Z, Zhan S, Kong Y, et al. Applied value of cranial brain imaging quality of high-field strong head flexible coil. Radiol Pract. 2020;35(04):555-559.

\section{Publish your work in this journal}

Neuropsychiatric Disease and Treatment is an international, peerreviewed journal of clinical therapeutics and pharmacology focusing on concise rapid reporting of clinical or pre-clinical studies on a range of neuropsychiatric and neurological disorders. This journal is indexed on PubMed Central, the 'PsycINFO' database and CAS, and is the official journal of The International Neuropsychiatric Association (INA). The manuscript management system is completely online and includes a very quick and fair peer-review system, which is all easy to use. Visit http://www.dovepress.com/testimonials.php to read real quotes from published authors. 